\title{
Papers
}

\section{Self reported stressful life events and exacerbations in multiple sclerosis: prospective study}

\author{
D Buljevac, W C J Hop, W Reedeker, A C J W Janssens, F G A van der Meché, P A van Doorn, \\ R Q Hintzen
}

\begin{abstract}
Objective To study the relation between self reported stressful life events not related to multiple sclerosis and the occurrence of exacerbations in relapsing-remitting multiple sclerosis.

Design Longitudinal, prospective cohort study. Setting Outpatient clinic of department of neurology in the Netherlands.

Participants Patients aged 18-55 with relapsing-remitting multiple sclerosis, who could walk with a cane or better (score of 0-6.0 on the expanded disability status scale), and had had at least two exacerbations in 24 months before inclusion in the study. Patients with other serious conditions were excluded.
\end{abstract}

Main outcome measure The risk of increased disease activity as measured by the occurrence of exacerbations after weeks with stressful events. Results Seventy out of 73 included patients (96\%) reported at least one stressful event. In total, 457 stressful life events were reported that were not related to multiple sclerosis. Average follow up time was 1.4 years. Throughout the study, 134 exacerbations occurred in 56 patients and 136 infections occurred in 57 patients. Cox regression analysis with time dependent variables showed that stress was associated with a doubling of the exacerbation rate (relative risk 2.2, 95\% confidence interval 1.2 to $4.0, \mathrm{P}=0.014$ ) during the subsequent four weeks. Infections were associated with a threefold increase in the risk of exacerbation, but this effect was found to be independent of experienced stress.

Conclusion Stressful events were associated with increased exacerbations in relapsing-remitting multiple sclerosis. This association was independent of the triggering effect of infections on exacerbations of multiple sclerosis.

\section{Introduction}

Multiple sclerosis is a chronic progressive disease that is characterised by a course of exacerbations and remissions in $85-90 \%$ of patients. Exacerbations are a clinical reflection of increased inflammatory activity in the central nervous system. ${ }^{12}$ They occur in an unpredictable fashion, but the consensus is that they are mediated through immunological mechanisms. Precipi- tating factors that have been identified are infections (of the upper respiratory tract) and the early postpartum period. ${ }^{3-5}$ Psychological stress is an additional factor that has been implicated repeatedly as a determinant of disease activity ever since Charcot first described the disease $^{6-13}$ The biological rationale for such an effect is that psychological stress can modulate immunological mechanisms via the hypothalamic-pituitary-adrenal axis and the sympathetic nervous system. ${ }^{145}$ However, evidence on the relation between psychological stress and exacerbations of multiple sclerosis is limited, partly because of the design of previous studies and the lack of agreement about the definition of stress. The recent report of the American Academy of Neurology about the relation between stress and exacerbations of multiple sclerosis stressed the need to obtain tightly defined prospective data ${ }^{16}$ We assessed in a prospective cohort setting whether life events that are perceived as stressful by patients themselves are associated with exacerbations in relapsing-remitting multiple sclerosis. Moreover, we investigated whether stressful events and infections are independently associated factors.

\section{Patients and methods}

This study was part of the Rotterdam study on exacerbations that was conducted as a single centre study between July 1997 and December 1999. ${ }^{4}$ Included were patients aged 15-55 who had clinically definite multiple sclerosis with a relapsing-remitting course. All patients gave written consent before participating in the study.

\section{Study design}

We collected clinical data prospectively during regular and additional visits at the outpatient clinic. Regular visits were scheduled every eight weeks. Additional visits were arranged within three days after patients reported symptoms of either infection or exacerbation. Each of these additional visits was followed by a control visit three weeks later. Neurological examination was performed at every regular and additional visit, and the same investigator (DB) scored the expanded disability status scale. Every Sunday during follow up patients assessed stressful events in self reported weekly diaries. Patients were asked whether they had experienced an emotionally stressful event in the preceding week. If they had they were asked to describe the event in their
Department of Neurology, Erasmus MC, Postbox 2040, $3000 \mathrm{CA}$ Rotterdam, Netherlands D Buljevac medical doctor W Reedeker medical student F G A van der Meché neurologist P A van Doorn neurologist R Q Hintzen neurologist Department of Epidemiology and Biostatistics, Erasmus MC W C J Hop biostatistician Department of Medical Psychology and Psychotherapy, Erasmus MC A C J W Janssens psychologist Correspondence to: R Q Hintzen rhintzen@xs4all.nl

bmj.com 2003;327:646 
Table 1 Baseline characteristics of 16 male and 56 female patients with multiple sclerosis who were included in the study. Values are means (medians, ranges) unless otherwise specified

\begin{tabular}{lc} 
Characteristic & Value \\
\hline Mean age (years) & 39.9 (median 40, range 19-55) \\
\hline Duration of disease (years since diagnosis) & 5.2 (median 4, range 1-25) \\
\hline Expanded disability status score at baseline & 2.6 (median 2.0, range 0-6.0) \\
\hline Diaries completed (weeks) & $5416^{*}$ \\
\hline No of exacerbations & 134 \\
\hline No of infections & 136
\end{tabular}

*84\% of total follow up.

own words. The diaries were collected at each regular visit. Two neurologists (DB and $\mathrm{RQH}$ ) independently evaluated whether the reported stressful events were directly connected to existing signs or symptoms of multiple sclerosis, to exclude stress caused by multiple sclerosis itself.

This was done blinded for the time point that the event was noted. Any differences were discussed with a third investigator (PAvD), until a final evaluation was reached by consensus.

An exacerbation was defined as a worsening of existing symptoms or appearance of new symptoms, lasting more than 24 hours and after at least 30 days of improvement or stability. ${ }^{17}$ Patients reported suspected exacerbations, which were then confirmed at additional visits. Neurological deterioration only temporarily associated with a period of fever was not considered as an exacerbation. Infection was defined as the appearance of coryza, sore throat, flu-like feeling, myalgia, fever, diarrhoea, or a urinary infection lasting for more than 24 hours.

\section{Statistical analysis}

Mohr et al thought that increased stress affects inflammatory lesions observed on magnetic resonance imaging within a few weeks. ${ }^{18}$ Other known precipitating factors of disease activity of multiple sclerosis act within several weeks. For example, infections increase the risk of exacerbation until five weeks after onset, ${ }^{3}$ and the effect of the early postpartum period can be observed within four weeks after delivery. ${ }^{5}$ We therefore chose a window of four weeks to study the association between stress and exacerbations.

After each stressful event patients were considered to enter a period of four weeks with high risk of an exacerbation. (For example, if a patient reported stressful events during the 20th, 30th, and 33rd week of follow up, the first high risk period ranged from week 21

Table 2 Categories of reported stressful events

Event

No of times reported $(n=457)$

Illness or problems with close family member

\begin{tabular}{ll} 
IIIness or problems with close family member & 107 \\
\hline Job stress & 54 \\
\hline Events related to house or car (repair, theft, etc) & 52 \\
\hline Sudden personal discomfort, not related to multiple sclerosis ${ }^{*}$ & 52 \\
\hline Death of distant relative or friend & 35 \\
\hline Financial problems & 31 \\
\hline Death of close family member & 29 \\
\hline Illness or problems with distant relative or friend & 28 \\
\hline IIness or death of pet & 27 \\
\hline Problems in relationship or marriage & 24 \\
\hline Stress related to holiday & 18
\end{tabular}

*Encompasses pain (gastric, dental, headache), physical trauma, minor surgery, and occurrence of sudden negative emotions. to week 24 . The second high risk period for this patient started in week 31 and lasted until week 37 because of the extension of the period by the event at week 33.)

To assess the predictive value of the periods of high risk, at each point in time during follow up we compared the exacerbation rates of patients at high risk and patients not at high risk. We used Cox regression with time dependent variables separately for the first, second, and third exacerbation. ${ }^{19}$ In analysing the second and third exacerbation we considered the time axis to originate at the time of the previous exacerbation. In relation to infections we defined another (time dependent) period at risk, which extended from two weeks before the onset of an infection until five weeks thereafter. ${ }^{3}{ }^{4}$ We used multivariate Cox regression to evaluate simultaneously the associations of the high risk period due to stressful events and due to infections. We included appropriate interaction terms to evaluate whether or not both factors were independently associated with the exacerbation rate. We used Logxact (Cytel Software Corporation, Cambridge, MA, USA) for calculations for this particular analysis. Results are expressed as relative exacerbation rates, and $\mathrm{P}=0.05$ (two sided) was considered the level of significance.

\section{Results}

\section{Patients and reported stressful life events}

We identified 110 patients as eligible for inclusion. Owing to the intense schedule of the study, 37 patients chose not to take part. Table 1 shows baseline characteristics of the participating patients. Seventy out of the 73 included patients (96\%) reported at least one stressful event. Average follow up time for the study group was 74 weeks (median 88, range 8-120) or 1.4 years. Thirteen patients dropped out before the end of the study, but they still participated for a median of 32 weeks (mean 46, range 8-112) per individual.

We found no relation between the cumulative number of reported stressful events and the probability of dropping out of the study. In total we analysed 124 patient years. The diaries that had been completed properly covered $84 \%(5416 / 6466)$ of all weeks of the total follow up time, and these were all included in the analysis. In total 505 stressful life events were reported. Forty eight events were directly related to signs or symptoms of multiple sclerosis and were excluded, which left 457 events for analysis. Table 2 shows a categorisation of the events. The average duration of a stressful event as noted in the diaries was 2.8 weeks. We assessed mental health at entry into the study by mental health subscores of the quality of life scale of the short form questionnaire (SF-36). The average score was 68.6 (SD 17.4), which was comparable with the scores that we observed in an independent study population of 101 patients with relapsing-remitting multiple sclerosis. ${ }^{20}$ Overall, 134 exacerbations occurred in 56/73 patients-an average of 1.3 exacerbations per year. Altogether 136 episodes of infections were seen in 57 patients-an average of 1.3 infections per year.

Stressful events and the risk of exacerbation For each week of the study we grouped patients according to whether or not they had experienced 


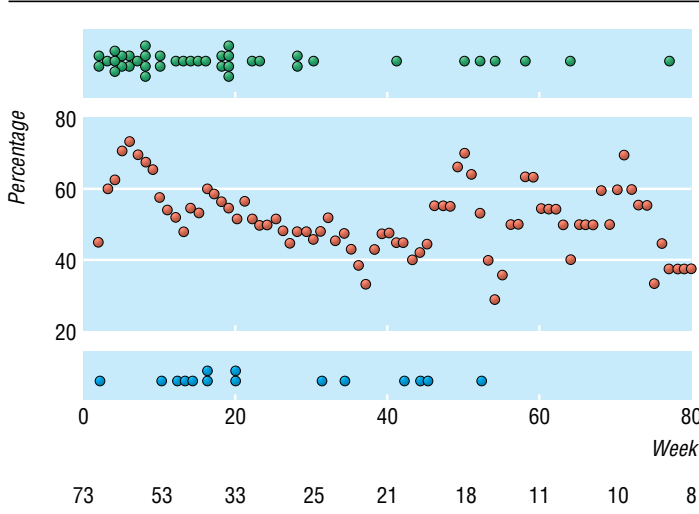

Fig 1 Presence of reported stress during the time window of four weeks before the first exacerbation.

stress in the four weeks immediately preceding it. Figure 1 shows the prevalence of stress for patients at risk of a first exacerbation in relation to the week of the study, and also shows the presence of stress in the preceding four weeks for individual patients with an exacerbation $(n=55)$. It shows that, although the prevalence of self reported stressful events remains more or less constant at about $50 \%$, most patients $(40 / 55)$ with an exacerbation had experienced stress during the four preceding weeks (one exacerbation occurring in week 1 could not be evaluated for stress in preceding weeks and was excluded from the analysis).

Figure 2 (left panel) shows the cumulated risks for exacerbation according to whether (curve A) or not (curve B) stress has been present in the four preceding weeks. The right panel shows the cumulated risk, but now after a first exacerbation had occurred. From the curves (log scaling) it can be concluded that the risk for occurrence of exacerbation associated with a four week period with one stressful event in it was about double after four weeks without reported stress. Cox univariate regression showed that a stressful event is associated with 2.2 times $(95 \%$ confidence interval 1.2 to 4.0 , $\mathrm{P}=0.012)$ the rate of exacerbation during the following four weeks. The same analysis for the second exacerbation gave similar results (fig 2, right panel). The risk for the second exacerbation was increased 2.7 times (1.2 to $5.9, \mathrm{P}=0.015)$ after a four week period with one stressful event. We found no significantly increased risk in relation to stress for third exacerbations (relative risk 1.2, $\mathrm{P}=0.62$ ), but the $95 \%$ confidence interval was wide (0.5 to 3.0$)$.
We compared the risk of exacerbation after four weeks with a single reported stressful event with that of four weeks with multiple reported stress events and found no significant differences. This indicates that the effect of stress on exacerbations is not "dose dependent."

\section{Infections, stressful events, and exacerbations}

Infections are associated with an increased risk of exacerbations of multiple sclerosis. ${ }^{3}{ }^{4}$ Table 3 shows the results of a simultaneous evaluation by using multivariate Cox regression of both reported stress and the presence of an increased risk of exacerbation around the time of clinical infection ("at risk period"). For the first two exacerbations, both evaluated factors were significant risk factors, whereas for the third exacerbation only the presence of infections was significantly related to this outcome.

Further analysis showed that both risk factors did not significantly depend on each other. The fact that these two factors were independently associated with the course of the disease implies that the risk for the onset of the first exacerbation in patients with both of these factors is increased up to 6.4 times $(2.2 \times 2.9)$ compared with patients in whom both factors are absent.

\section{Discussion}

In patients with multiple sclerosis the experience of at least one stressful event during a period of four weeks was associated with double the risk of an exacerbation within the next week.

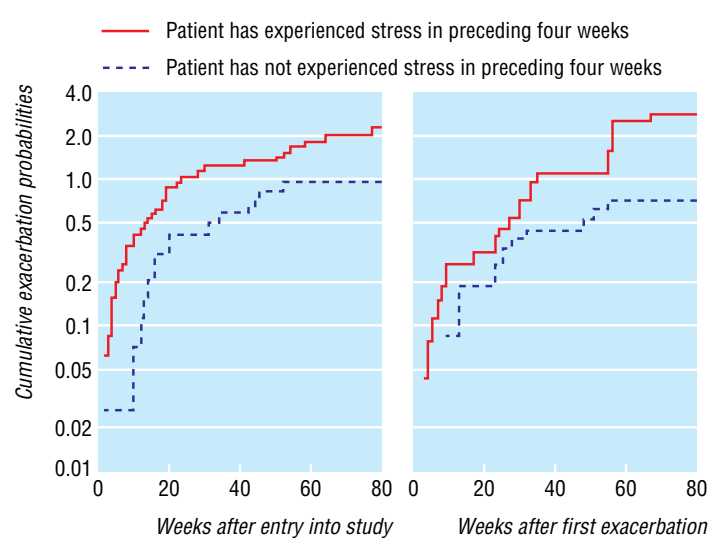

Fig 2 Cumulative probability for the onset of the first and second exacerbation

Table 3 Multivariate analysis of the risk of occurrence of exacerbation with respect to the presence of stress and infection

\begin{tabular}{|c|c|c|c|c|c|}
\hline \multirow[b]{2}{*}{ Exacerbation } & \multirow[b]{2}{*}{ Risk factor } & \multirow[b]{2}{*}{ Relative risk (95\% CI) } & \multicolumn{2}{|c|}{ No of exacerbations/No of weeks of follow up } & \multirow[b]{2}{*}{$P$ value } \\
\hline & & & At high risk & At low risk & \\
\hline \multirow[t]{2}{*}{ First $(\mathrm{n}=55)$} & Stress $^{*}$ & $2.2(1.2$ to 4.0$)$ & $40 / 1143$ & $15 / 984$ & 0.014 \\
\hline & Infection** & $2.9(1.6$ to 5.1$)$ & $20 / 365$ & $36 / 1866$ & $<0.001$ \\
\hline \multirow[t]{2}{*}{ Second $(n=32)$} & Stress & $2.7(1.2$ to 6.0$)$ & 20/762 & $12 / 1130$ & 0.015 \\
\hline & Infection & 2.7 (1.1 to 6.3 ) & $8 / 253$ & 24/1705 & 0.023 \\
\hline \multirow[t]{2}{*}{ Third $(n=23)$} & Stress & $1.0(0.4$ to 2.5$)$ & $12 / 337$ & $10 / 352$ & 0.99 \\
\hline & Infection & $3.2(1.3$ to 8.1$)$ & 9/157 & $14 / 568$ & 0.014 \\
\hline
\end{tabular}

Owing to missing diary data weeks could occasionally not be classified for stress (high or low risk). The number of weeks is therefore not always the same for both risk factors (stress and infection).

${ }^{*}$ Reported at least once during the preceding four weeks.

${ }^{*}$ *Week is part of period at risk for infection. Each time a stressful event occurs a period with high risk starts that lasts four weeks. With respect to infections, a

period with high risk starts two weeks before the onset of an infection and ends five weeks after the infection. Relative risk represents the relative risk of an exacerbation during these high risk periods. 
Relation to previous studies

An association between stressful life events and disease activity in multiple sclerosis has been assumed for a long time, ${ }^{7-14}$ although negative findings have been reported. ${ }^{21}$ Several methodological difficulties ${ }^{16}$ have hampered a clear conclusion on this issue. ${ }^{7-1322}$ For example, most studies had a retrospective design that probably introduced recall bias. In the available studies, exacerbations were often not confirmed, and the intervals for recording of stress or stress events were within the range of months to even years. In our study, stressful life events were reported weekly by patients themselves and recorded in a prospective fashion. Patients were seen at regular survey visits at close intervals, and additional visits were arranged in cases of infection or exacerbation. ${ }^{4}$ All relapses were confirmed by neurological examination by a doctor. We focused on either the mere presence or absence of personal experience of a stressful event as we judged this the most practical approach to analyse the external stress that a patient faces during daily life. We made maximum efforts to exclude interference by situations of stress directly originating from the symptoms of the disease itself. Internal robustness of the results is found in the fact that increased risks were also observed for a second exacerbation. This significance was lost for a third exacerbation, possibly because of the small numbers; the resulting confidence interval is very wide. One study analysed the relation between stress and disease activity by using a surrogate marker instead of clinical exacerbations. ${ }^{18}$ Mohr et al showed increased odds of developing new gadolinium enhancing lesions in the weeks after moderately stressful life events. They found, however, no relation between the stress events and clinical exacerbations, which were stringently defined as changes of 1.0 point or more on the expanded disability status scale. An important difference to our study is that we used the criteria of Schumacher et al-new or worsening symptoms associated with a confirmatory change lasting more than 24 hours, in the absence of fever. ${ }^{17}$

For the analysis, on a priori grounds, ${ }^{3,518}$ we had chosen a period with increased risk of four weeks after a stressful event. To investigate the sensitivity of our findings with respect to this choice, we also analysed the exacerbation rates for some other time windows. When we repeated the analysis with respective windows of two, three, and five weeks, only the window of three weeks showed a significantly increased risk of exacerbation (relative risk 2.1, $\mathrm{P}=0.012$ ). We obtained similar results when considering these windows before a second exacerbation. We did not, however, record whether the events took place at the beginning, the middle, or the end of the week. Therefore the period of increased risk is probably three to five weeks.

\section{Limitations of this study}

We collected participants' diaries every eight weeks. Although this included frequent contact with patients and extensive control for compliance,the diaries may not have been filled immediately after an event happened but rather, for example, just before regular appointments or around major events. However, we found no indication for reporting around such biased time points as no increased stress in relation to infections was reported in the week of the relapse itself

\section{What is already known on this topic}

Psychological stress has been implicated as a determinant of disease activity in multiple sclerosis

Evidence on the relation between stressful events and exacerbations of multiple sclerosis is lacking

A recent report of the American Academy of Neurology emphasised the need to obtain tightly defined prospective data

\section{What this study adds}

Patients with multiple sclerosis who experience a stressful event are subsequently at increased risk of an exacerbation of their disease

Stress and infection are independently associated with the risk of an exacerbation

nor just before the regular eight week visits. Even if this had been the case it would have resulted in an underestimation of the stress during the four weeks preceding exacerbations.

Furthermore, we relied on a single item, self reported stress scale instead of using a validated multiitem instrument. Our stress scale assessed the perceived presence or absence of psychological stress but provided no information on the intensity of stress and did not use a normative definition of stressful events. We anticipated that compliance in the frequent and prospective data sampling would be maintained by the use of a single item rather than a multi-item stress scale. Such an approach is similar to previous studies on infections and exacerbations that also analysed the mere occurrence of infections rather than taking into account the severity or exact character of infection. ${ }^{3}$ An important difference to other studies ${ }^{7-13} 162122$ is our emphasis on self report of exposure to stress events as well as the frequent and prospective sampling.

\section{Possible mechanisms}

The mechanism behind increased stress and the induction of inflammatory disease activity in an autoimmune disease such as multiple sclerosis is not yet fully understood. Modulation of both the hypothalamic-pituitary-adrenal axis and the sympathetic system can have a notable effect on lymphocyte dependent inflammatory reactions, at least partly by modulation of cytokine production and lymphocyte expressed adrenergic receptors. ${ }^{14} 152324$ In future clinical studies on this issue it would be of interest to measure neuroendocrine variables in blood samples, such as adrenaline, cortisol, and lymphocyte expression of neuroendocrine receptors. This could also serve as an objective biological measure of stress. However, for such a study it would be necessary to obtain blood samples frequently.

Stress and infection and the risk of an exacerbation Certain types of psychological stress can suppress immune reactions, and this could lead to increased susceptibility to infections. ${ }^{25}$ Therefore our observed association of stressful events with exacerbations of multiple sclerosis might simply be linked to an increase of infections after stressful events in our patients. How- 
ever, we found no evidence for an increase of infections after stressful life events. Stress and infection were independently associated with the risk of exacerbation. It will not be easy to tackle these factors in a given patient, because both infections and stressful events cannot simply be eradicated from patients' lives. The knowledge that stressful events are associated with disease activity adds important information to the limited insight that patients and their caregivers have on this unpredictable disease.

We thank the late Monica van der Hoven, who performed secretarial and organisational tasks for this study, and D Dippel for critical reading of the manuscript.

Contributors: DB was responsible for planning and execution of the study. He was also responsible for patients' care, performed data collection and interpretation, and wrote the article together with RQH. RQH supervised the study. WR participated in data collection and interpretation and in writing of the first draft. WCJH performed the statistical analysis and contributed to writing. ACJWJ participated in setting up the study and writing. FGAvdM participated in setting up the study and interpretation of data. PAvD participated in setting up the study, caring for patients, data interpretation, and writing. $\mathrm{DB}$ and $\mathrm{RQH}$ are guarantors.

Funding: Stichting Vrienden MS Research, the Preventiefonds, and Erasmus MC Rotterdam.

Competing interests: None declared.

Ethical approval: The medical ethical committee of Erasmus MC approved the study.

1 Noseworthy JH, Lucchinetti C, Rodriguez M, Weinshenker BG. Multiple sclerosis. N Engl J Med 2000;343:938-52.

2 Compston A, Coles A. Multiple sclerosis. Lancet 2002;359:1221-31.

3 Sibley WA, Bamford CR, Clark K. Clinical viral infections and multiple sclerosis. Lancet 1985;:1313-5.

4 Buljevac D, Flach HZ, Hop WC, Hijdra D, Laman JD, Savelkoul HF, et al. Prospective study on the relationship between infections and multiple Prospective study on the relationship between

5 Confavreux C, Hutchinson M, Hours MM, Cortinovis-Tourniaire P Moreau T. Rate of pregnancy-related relapse in multiple sclerosis. $N$ Engl J Med 1998;339:285-91.

6 CharcotJM. Lectures on the diseases of the nervous system. In: Lectures on the diseases of the nervous system. London: New Sydenham Society, 1879:157-222.
7 Warren S, Warren KG, Cockerill R. Emotional stress and coping in multiple sclerosis (MS) exacerbations.J Psychosom Res 1991;35:37-47.

8 Grant I, Brown GW, Harris T, McDonald WI, Patterson T, Trimble MR. Grant I, Brown GW, Harris T, McDonald WI, Patterson T, Trimble MR.
Severely threatening events and marked life difficulties preceding onset or exacerbation of multiple sclerosis. J Neurol Neurosurg Psychiatry $1989 ; 52: 8-13$

9 Sibley WA. Risk factors in multiple sclerosis. In: Raine CS, McFarland HF, Tourtelotte WW, eds. Multiple sclerosis: clinical and pathogenetic basis. London: Chapman and Hall, 1997:141-8.

10 Warren S, Greenhill S, Warren KG. Emotional stress and the development of multiple sclerosis: case-control evidence of a relationship. J Chronic Dis 1982;35:821-31.

11 Franklin GM, Nelson LM, Heaton RK, Burks JS, Thompson DS. Stress and its relationships to acute exacerbations in multiple sclerosis. J Neurol Rehabil 1988;2:7-11.

12 Schwartz CE, Foley FW, Rao SM, Bernardin LJ, Lee H, Genderson MW. Stress and course of disease in multiple sclerosis. Behav Med 1999;25:110-6.

13 Kroencke DC, Denney DR. Stress and coping in multiple sclerosis: exacerbation, remission and chronic subgroups. Mult Scler 1999;5:89-93.

14 Chelmicka-Schorr E, Arnason BG. Nervous system-immune system interactions and their role in multiple sclerosis. Ann Neurol 1994;36(suppl):s29-32.

15 Sternberg EM. Neuroendocrine regulation of autoimmune/ inflammatory disease. J Endocrinol 2001;169:429-35.

16 Goodin DS, Ebers GC, Johnson KP, Rodriguez M, Sibley WA, Wolinsky JS. The relationship of MS to physical trauma and psychological stress: The relationship of MS to physical trauma and psychological stress:
report of the Therapeutics and Technology Assessment Subcommittee of report of the Therapeutics and Technology Assessment Subcommitee
the American Academy of Neurology. Neurology 1999;52:1737-45.

17 Schumacher GA, Beebe G, Kibler RF, Kurland LT, Kurtzke JF, McDowell F, et al. Problems of experimental trials of therapy in multiple sclerosis: report by the panel on the evaluation of experimental trials of therapy in multiple sclerosis. Ann N Y Acad Sci 1965;122:552-68.

18 Mohr DC, Goodkin DE, Bacchetti P, Boudewyn AC, Huang L, Marrietta $\mathrm{P}$, et al. Psychological stress and the subsequent appearance of new brain MRI lesions in MS. Neurology 2000;55:55-61.

19 Cox DR. Regression models and life-tables. J Roy Stat Soc B 1972;34:187220.

20 Janssens ACJW, Van Doorn PA, de Boer JB, van der Meché FGA, Passchier J, Hintzen RQ. Impact of recently diagnosed multiple sclerosis on quality of life, anxiety, depression and distress of patients and partners. Acta Neurol Scand 2003 (in press).

21 Rabins PV, Brooks BR, O'Donnell P, Pearlson GD, Moberg P, Jubelt B, et al. Structural brain correlates of emotional disorder in multiple sclerosis. Brain 1986;109:585-97.

22 Nisipeanu P, Korczyn AD. Psychological stress as risk factor for exacerbations in multiple sclerosis. Neurology 1993;43:1311-2.

23 Jafarian-Tehrani M, Sternberg EM. Animal models of neuroimmune interactions in inflammatory diseases. J Neuroimmunol 1999;100:13-20.

24 Antel JP, Owens T. Immune regulation and CNS autoimmune disease. J Neuroimmunol. 1999;100:181-9.

25 Sheridan JF, Dobbs C, Brown D, Zwilling B. Psychoneuroimmunology: 5 Sheridan JF, Dobbs C, Brown D, Zwilling B. Psychoneuroimmunology:
stress effects on pathogenesis and immunity during infection. Clin Microbiol Rev 1994;7:200-12.

(Accepted 17 July 2003) 